\title{
Fiabilidad y validez de la versión abreviada en español del Cocaine Craving Questionnaire-Now (CCQ-N-10)
}

\section{Reliability and validity of the Spanish adaptation of the brief form of the Cocaine Craving Questionnaire-Now (CCQ-N-10)}

loseba Iraurgi Castillo'; Joan Trujols Albet²; Juan Manuel Jiménez-Lerma ${ }^{3}$; Miguel Landabaso ${ }^{4}$
${ }^{1}$ Módulo de Asistencia Psicosocial de Rekalde, Bizkaia

2 Unidad de Conductas Adictivas del Hospital de la Santa Cruz y San Pablo, Barcelona

${ }^{3}$ Centro de Salud Mental de Arrasate, Gipuzkoa

${ }^{4}$ Centro de Tratamiento de Toxicomanías de Barakaldo, Bizkaia

Enviar correspondencia a:

loseba Iraurgi Castillo. Módulo Psicosocial de Rekalde. Camilo Villabaso 24 lonja. 48002 Bilbao. E-mail: iraurgi@telefonica.net

\section{RESUMEN}

Objetivo: Valorar la calidad métrica de la versión abreviada de 10 items del Cocaine Craving Questionnaire-Now (CCQ-N-10) en su adaptación al castellano. Métodos: La muestra está constituida por 63 personas que inician tratamiento por abuso $(n=23)$ o dependencia de cocaina $(n=40)$ según criterios DSM-IV. Se administró una batería de instrumentos entre los cuales se hallan la versión extendida de 45 items del Cocaine Craving Questionnaire-Now (CCQ-N-45) de la que se extrae la versión abreviada, la Cocaine Craving Scale (CCS), una Escala Visual-Analógica del craving percibido (EVA), la Entrevista de Severidad de la Dependencia (SDS) y la Impresión Clínica Psiquiátrica (ICP).

Resultados: Todos los instrumentos muestran una alta consistencia interna (alpha de Cronbach para CCQ-N-10=0,95; CCQ $-\mathrm{N}-45=0,88 ; \operatorname{SDS}=0,79$ y $\mathrm{CCS}=0,93)$ y un Coeficiente de Correlación Intraclase para el test-retest del CCQ$\mathrm{N}-10$ de $0,59(\mathrm{p}<0,001)$. El análisis factorial del $C C Q-\mathrm{N}-10(\mathrm{KMO}=0,85)$ muestra unidimensionalidad llegando a explicar el 68,6\% de la varianza, con saturaciones entre 0,64 y 0,92. Un análisis factorial confirmatorio ratifica la unidimensionalidad observada ( $\mathrm{CFI}=0,91 ; \mathrm{SRMR}=0,06$ ). El CCQ- $\mathrm{N}-10$ explica un $86,6 \%$ de la varianza de la versión extendida (CCQ-N-45) y presenta validez convergente con otros constructos afines (correlación CCQ-CCS $r=0,64$; CCQ-EVA $r=0,65$; CCQ-SDS $r=0,53$; y CCQ-ICP $r=0,50)$. Se observan diferencias en las puntuaciones del CCQ$\mathrm{N}-10(F=7,16 ; p=0,010)$ cuando es administrado a personas con diagnóstico de abuso $(M=2,76)$ respecto a las personas diagnosticadas de dependencia $(M=3,62)$. Conclusiones: La adaptación española del CCQ-N-10 presenta adecuación métrica y puede considerarse como un instrumento oportuno para su utilización en dependencia de cocaina.

Palabras clave: Deseo de cocaina, cuestionario, adaptación transcultural, propiedades psicométricas, Evaluación.

\section{ABSTRACT}

Objective: To assess the metric properties of the abridged, 10-item version of the Cocaine Craving Questionnaire-Now (CCQ-N-10) in its Spanish adaptation. Method: The sample consists of 63 people beginning treatment for cocaine-abuse $(n=23)$ or dependence $(n=40)$ disorders according to DSM-IV criteria. Participants completed an assessment battery that included the extended, 45 -item version of the Cocaine Craving Questionnaire (CCO-N-45) -in which the CCQ-N-10 is embedded-, the Cocaine Craving Scale (CCS), a Visual Analog Craving Scale (VAS), the Severity of Dependence Scale (SDS), and the Clinical Psychiatric Impression (CPI).

Results: Cronbach's values for the CCQ-N-10, CCQ-N-45, SDS and CCS scales were $0.95,0.88,0.79$ and 0.93 , respectively, indicating that all instruments show high internal consistency. Test-retest reliability for the CCQ-N-10 was fair (Intraclass Correlation Coefficient $=0.59 ; \mathrm{p}<0.001)$. A factor analysis of the $\mathrm{CCQ}-\mathrm{N}-10$ $(\mathrm{KMO}=0.85)$ shows a one-factor structure explaining $68.6 \%$ of the variance, with factor loadings ranging from 0.64 to 0.92 . Confirmatory factor analysis supports the unidimensionality of the scale ( $C F=0.91 ; S R M R=0.06)$. The $C C Q-N-10$ explains $86.6 \%$ of the variance accounted for by the CCQ-N extended version (CCQ-N-45) and shows convergent validity with related measures such as the CCS $(r=0.64$, $p<0.001)$, VAS $(r=0.65, p<0.001), \operatorname{SDS}(r=0.53, p<0.001)$ and $C P I(r=0.50, p<0.001)$. Persons fulfilling criteria for cocaine-dependence disorder, compared to those who present cocaine abuse, show higher CCQ-N-10 scores $\left(F_{(1,60.6)}=7.16, p=0.010\right)$. Conclusions: The Spanish adaptation of the CCQ-N-10 shows metric adequacy, and can be considered as a suitable assessment instrument in relation to cocaineuse disorders.

Key words: Cocaine craving, questionnaire, cross-cultural adaptation, psychometric properties, assessment. 


\section{INTRODUCCIÓN}

El craving, entendido como el deseo de experimentar los efectos de una sustancia psicoactiva que ha sido probada previamente ${ }^{1,2}$, se ha convertido en las últimas décadas en un concepto controvertido pero de gran importancia en el ámbito de las adicciones, tanto desde el campo de la investigación como desde su perspectiva clínica. A pesar de los múltiples modelos explicativos del fenómeno y no existir un consenso sobre su definición ${ }^{3}$, sí hay coincidencia entre los clínicos y entre los propios usuarios sobre el papel relevante que ocupa el craving en el proceso de adicción y en su vivencia cotidiana ${ }^{4-6}$.

La valoración del craving asociado al consumo de cocaina viene realizándose en el ámbito clínico y de investigación a través de instrumentos de autoinforme, habiéndose desarrollado hasta el momento un repertorio aceptable $e^{3,7}$ entre los cuales destacan la Voris Cocaine Craving Scale ${ }^{8}$-VCCS-; el Cocaine Craving Questionnaire ${ }^{9}$-CCQ-, o la Cocaine Craving Scale $e^{10-11}$-CCS-. Todos ellos muestran unas adecuadas caracteristicas psicométricas pero, entre ellos, el CCO es el que ha mostrado una mayor validez de contenido con un sólido soporte teórico en la base de su construcción ${ }^{12-14}$. A pesar de la robustez del $\mathrm{CCO}$, una de las limitaciones más importantes de dicho instrumento es su extensión, 45 items, lo cual convierte el CCO en un instrumento bueno pero no óptimo para su utilización en el ámbito clínico.

Para subsanar esta limitación, diversos grupos de investigación han propuesto y validado diferentes versiones breves del $\mathrm{CCQ}^{15-18}$. En nuestro ámbito, Muñoz Garcia ${ }^{15-16}$ ha desarrollado una versión de 12 items (el CCQ-G-12) ${ }^{17}$ a partir del formato de valoración general del CCQ-45 adaptado al castellano por Tejero, Trujols y Siñol ${ }^{7}$, obteniendo un instrumento con una elevada consistencia interna que consigue reproducir satisfactoriamente cuatro de las dimensiones teóricas propuestas por Tiffany ${ }^{9,12-14}$, y presenta una adecuada validez discriminante; si bien la adaptación de dicho instrumento nace en el contexto de la investigación básica ${ }^{15}$. Desde un ámbito más asistencial, y con el objetivo de obtener una medida que sirviera para la valoración clínica del craving, el grupo de Sussner ${ }^{17}$ desarrolló el CCQ-N-10 extrayendo los 10 items que, en el proceso de desarrollo de la versión original del CCO-Now ${ }^{9}$, presentaban un mayor peso factorial en el primer factor de dicho instrumento ('deseo de consumir'). El CCQ-N-10 está diseñado pues para proporcionar una única puntuación del constructo, planteándose la unidimensionalidad del instrumento ${ }^{17}$. Los resultados de dos estudios de validación ${ }^{17-18}$ del CCO-N-10 muestran que dicho cuestionario presenta una elevada consistencia interna (alpha de Cronbach de 0,90 y 0,87, respectivamente) y una alta correlación con la puntuación total de la versión de 45 ítems del CCQ-N -CCQ-N-45- ( $r=0,85$ y 0,87, respectivamente). El $\mathrm{CCO}-\mathrm{N}-10$ presenta igualmente una elevada correlación con la versión del CCQ una vez eliminados los diez ítems extraídos -CCO-N-35- $(r=0,78)^{17}$, una adecuada validez convergente con otras medidas del craving $\left(r=0,47^{17}\right.$ con el item de craving de la VCCS y $r=0,46^{18}$ tanto con el item de intensidad como con el de frecuencia del craving de la escala Cocaine
Selective Severity Assessment) y una adecuada validez predictiva (el riesgo de recaída aumenta un 3\% por cada punto incremental en la escala) ${ }^{18}$. Todos estos resultados presentan el CCQ-N-10 como un instrumento con buenas caracteristicas métricas para la valoración del craving de cocaína que, por su brevedad, resulta muy versátil para su utilización en la práctica clínica habitual.

El CCQ, tanto en su versión 'general' (CCQ-G) como en la 'actual' (CCQ-N), ha sido traducido al castellano por Tejero, Trujols y Siñol', pero hasta el momento no se han ofrecido datos publicados sobre las características psicométricas de ninguna de las dos versiones del instrumento adaptado. Ante la necesidad de instrumentos breves para la valoración del craving de cocaína en el ámbito clínico y dado que hasta la fecha únicamente disponemos de datos sobre las caracteristicas psicométricas de la versión breve española del CCQ-G $(\mathrm{CCO}-\mathrm{G}-12)^{16}$, el presente estudio tiene como objetivo explorar la validez y fiabilidad de la versión abreviada del CCO-N (CCO-N-10) en su adaptación al español, y valorar la convergencia de dicha versión respecto a la versión extendida (CCQ-N-45).

\section{MÉTODO}

\section{Participantes}

La muestra está formada por 63 personas, 53 hombres $(84,1 \%)$ y 10 mujeres $(15,9 \%)$, que iniciaban tratamiento por problemas adictivos relacionados con el uso de cocaina en seis centros públicos de drogodependencias del País Vasco. La edad media es de 36,6 años (rango 20,8 a 52,7), siendo la duración media del tiempo de consumo antes de solicitar el tratamiento actual de 9,6 años (rango 1 a 24). Para el total de casos se constató diagnóstico de dependencia $(n=40$; $63,5 \%)$ y/o abuso de cocaína $(n=23 ; 36,5 \%)$ según criterios DSM-IV-TR.

Los participantes fueron seleccionados de forma consecutiva conforme iniciaban tratamiento en alguno de los centros colaboradores y previa solicitud de consentimiento informado. Los criterios de inclusión fueron: 1) solicitar voluntariamente tratamiento por presentar problemas de consumo de cocaína, 2) iniciar tratamiento o haberlo iniciado en las dos semanas previas a la participación en el estudio, 3) ser mayor de 18 años, y 4) dar consentimiento de participación en el estudio. Los criterios de exclusión fueron: 1) presencia 0 antecedentes de trastornos psicóticos, 2) presencia de otro trastorno por dependencia a sustancias psicoactivas distintas a la cocaina, y 3 ) incapacidad de responder a los instrumentos de evaluación.

\section{Procedimiento}

Este estudio de valoración psicométrica del CCO está subsumido en otro más amplio cuyo objetivo es la valoración de distintas alternativas terapéuticas utilizadas bajo un encuadre naturalístico con seguimiento a 6 meses y evaluaciones quincenales ${ }^{19}$. Los datos utilizados en el presente es- 
tudio corresponden a los obtenidos en la línea base (inicio de tratamiento) y a las 2-3 semanas (para la valoración de la fiabilidad re-test).

Todos los sujetos participantes contestaban a una batería de cuestionarios (véase apartado de instrumentos) en presencia del clínico responsable de su tratamiento. Algunas escalas eran respondidas por los propios participantes (CCO-N-45, Escala Analógico Visual del Craving de la VCCS, Escala de Craving de Cocaína y Escala de Severidad de la Dependencia) y en el caso de dudas o dificultad para contestar eran ayudados por el clínico. Otros elementos de la batería de instrumentos (Impresión Clínica Global e Impresión Clínica Psiquiátrica) eran valorados por el propio personal asistencial atendiendo a las observaciones de la entrevista y su juicio clínico.

\section{Instrumentos}

Cuestionario de Craving de Cocaína (Cocaine Craving Questionnaire, $\mathrm{CCO})^{9,17}$. Para el presente estudio se ha utilizado la versión CCQ-Ahora (o CCO-Now, [CCO-N-45] en su acepción en inglés) adaptada al castellano por Tejero, Trujols y Siñol'. Está compuesta por 45 items de respuesta tipo Likert de 7 puntos en función del grado de acuerdo o de desacuerdo con los enunciados planteados. La versión abreviada, denominada CCQ-N-10, está constituida por 10 ítems extraídos del CCQ-N45 , cuyos enunciados y número de orden dentro del CCQ-N-45 aparecen al pie de la Tabla 1. Siguiendo el proceder de Sussner et al. ${ }^{17}$ se han calculado tres índices sumarios: 1) el correspondiente a la suma promediada de los 45 items del instrumento (CCO-N-45); 2) el que haría alusión a la versión abreviada de 10 items (CCQ-N-10), y 3) un último indice (CCQ-N-35) compuesto por los 35 items restantes del CCO-N-45 una vez extraídos los 10 que componen el CCQ-N-10. Los tres indices han sido calculados como la suma promediada, de modo que las puntuaciones oscilan entre un mínimo de 0 y un máximo de 6 puntos, de modo que a mayor puntuación mayor intensidad del deseo de consumir o craving. Previo al cálculo de la suma promediada, aquellos items planteados de forma inversa fueron recodificados invirtiendo el orden del valor señalado. En el caso del CCO-N-10 (Tabla 1), los items recodificados fueron los enunciados siguientes: 'Ahora creo que podria aguantarme sin tomar cocaína' y 'Ahora no tengo ningún deseo de tomar cocaína'.

Escala de Craving de Cocaina (Cocaine Craving Scale, CCS). La CCS ha sido desarrollada por Weiss et al. ${ }^{10-11}$ para la evaluación clínica del craving de cocaína y está compuesta por 5 ítems con respuesta en una escala de 0 a 9 puntos, ofreciendo un índice sumario promedio de modo que a mayor puntuación, mayor intensidad del craving. En el presente estudio, se ha empleado la adaptación española realizada por Tejero et al. ${ }^{7}$

Escala Analógico Visual del Craving (EVA-Craving). Tomando como referencia la Escala de Craving a Cocaina de Voris ${ }^{8}$, se diseñó una escala analógico visual de 100 milímetros para valorar 'el deseo o ganas de consumir durante las últimas 24 horas'. Se presentaba al entrevistado la escala graduada a modo de termómetro situándose en el extremo inferior o izquierdo el lema 'ningún deseo' y en el extremo superior o derecho el lema 'Incapaz de resistirme'; debiendo señalar con una raya aquel punto de la escala que representase su valoración del grado de deseo percibido. El resultado es una puntuación de escala continua entre 0 y 100 tomándose como valor la distancia medida con regla entre el extremo inferior o izquierdo y el punto de señalado por el entrevistado.

Escala de Severidad de la Dependencia (Severity of Dependence Scale, SDS). Esta escala fue desarrollada por el grupo de Gossop ${ }^{20-21}$ con el objetivo de medir los componentes psicológicos de la dependencia. Consta de cinco items tipo Likert con puntuaciones entre 0 y 4 que aportan una valoración sumaria que sitúa a las personas en un continuo según el nivel de gravedad de la dependencia (de menor a mayor). Adaptada al castellano por González-Saiz y Salvador-Carulla ${ }^{22}$, ha mostrado un adecuado comportamiento psicométrico en estudios de validación tanto nacionales como internacionales ${ }^{20-23}$.

Impresión Clínica Global (ICG) e Impresión Clínica Psiquiátrica (ICG) ${ }^{24-25}$. Se valoró la apreciación y juicio de los clínicos sobre la situación de los participantes a través de su valoración de experto tanto de los componentes globales de salud presentados (ICG) como del estado psiquiátrico (ICP). Para ello, en cada caso los clínicos respondieron a través de una escala de 8 puntos con rango 0-7, siendo las especificaciones de cada nivel las siguientes: 0 (no determinado), 1-2 (no del todo enfermo o dudosamente enfermo), 3 (ligero), 4 (moderado), 5 (grave), 6 (severo) y 7 (grado más extremo de la enfermedad).

Asimismo, se registraron datos sociodemográficos y de historia de consumo, entre los que se informó sobre el total de años de consumo y el número de días durante el último mes que habian consumido cocaina.

\section{Análisis}

Para el análisis de fiabilidad se ha calculado el coeficiente de consistencia interna alpha de Cronbach $(\alpha)$ para el total de la escala y para el resultado de la escala si se retirase alguno de sus ítems componentes. Asimismo, se ha calculado la correlación de cada item componente con el total de la escala obteniendo el coeficiente producto-momento de Pearson correspondiente. La fiabilidad test-retest se ha obtenido mediante el cálculo del Coeficiente de Correlación Intraclase $(\mathrm{CCl})$ entre la medida basal y un retest realizado a las 2-3 semanas.

Para valorar la validez de constructo del CCQ-N-10 se ha procedido a contrastar el modelo unifactorial, propuesto por los autores de la versión abrevida ${ }^{17}$, mediante la aplicación de dos procedimientos de analisis factorial (AF): exploratorio (AFE) y confirmatorio (AFC). En primer lugar, se realizó un análisis factorial de componentes principales con rotación varimax como estrategia exploratoria. Para comprobar el grado de interrelación de las variables se calculó el índice Kaiser-Meyer-Olkin (KMO) y la prueba de esfericidad de Barlett $\left(\chi^{2}\right)$. Se consideraron aquellos factores o dimensiones con valores propios (eigenvalue) superiores a 1, según el criterio de Kaiser. Para la interpretación de las dimensiones subyacentes se presentan las comunalidades y cargas facto- 
riales, considerándose aquellos ítems con saturación superior a 0,40. Secundariamente se realizó un AFC a partir de técnicas estructurales de covarianza con objeto de, en este caso, contrastar y/o verificar la unidimensionalidad del CCQ$\mathrm{N}-10$. Para ello, se estimó el grado de ajuste a los datos de la muestra del modelo teórico hipotetizado a través de la aplicación del programa EOS, utilizando para la estimación de los parámetros el método de máxima verosimilitud. Para evaluar el nivel de bondad de ajuste del modelo se han utilizado los siguientes indicadores: 1) Ji cuadrado de contraste de igualdad entre matrices [ha de ser no significativo $(p>0,05)$ o mostrar valores bajos; dado que este criterio suele estar afectado por el tamaño muestral, también han de tenerse en cuenta otros coeficientes], 2) la razón entre el Ji cuadrado y los grados de libertad del modelo $\left[\chi^{2} / g l\right.$, el cociente debe ser menor de 4; a menor índice, mejor ajuste], 3) el índice de bondad de ajuste $(\mathrm{GFI}), 4)$ el indice de ajuste comparativo de Bentler (CFI), 5) la raíz cuadrada media residual (RMSR), y 6) la raíz cuadrada media de error de aproximación (RMSEA). Para que exista un buen ajuste, los valores GFI y CFI deberían acercarse al valor 0,90 (cuanto mayor sea el valor, mejor es el ajuste), y los valores RMSR y RMSEA deberían ser menores o iguales a 0,05 (cuanto menor sea el valor, mejor ajuste). Asimismo, se ha calculado a partir de los coeficientes Lambda-X y Theta-Delta del AFC el coeficiente de fiabilidad del constructo y el porcentaje de varianza extractada ${ }^{26}$.

La validez convergente se ha estudiado a través del análisis de la asociación del CCO-N-10 con otros constructos afines (la EVA-Craving, la CCS, la SDS y la ICP e ICG), utilizando correlaciones producto momento de Pearson. La concordancia (validez concurrente) entre el CCO-N-10, el CCO-N-45 y $\mathrm{CCQ}-\mathrm{N}-35$ ha sido determinada a través del cálculo del coeficiente de correlación de Pearson ajustado (r), obtenido mediante modelos de regresión simple, ofreciéndose asimismo la estimación de la varianza explicada del CCQ-N-10 respecto al $\mathrm{CCO}-\mathrm{N}-45$ y al $\mathrm{CCO}-\mathrm{N}-35$ a través del coeficiente de determinación $\left(\mathrm{R}^{2}\right)$. La posible significación estadística de las diferencias entre las puntuaciones medias del CCO-N-10 respecto del CCO-N-45 ha sido estudiada a partir de la prueba t-test para datos apareados.

La validez discriminante se ha valorado mediante el contraste de diferencias en las puntuaciones del CCO-N-10 entre los dos subgrupos formados en función del tipo de trastorno adictivo diagnosticado: dependencia vs abuso de cocaína. Dado el incumplimiento de criterios paramétricos (Prueba de Levene: 14,$77 ; 1$ y 61 grados de libertad, $p<0,001$ ), se ha utilizado la prueba de Brown-Forsythe para su contraste.

Todos los análisis, salvo el análisis factorial confirmatorio, se han realizado mediante el programa SPSS V.11

\section{RESULTADOS}

En la Tabla 1 se presentan los principales datos descriptivos y de fiabilidad así como los principales resultados de los análisis factoriales exploratorio y confirmatorio de la escala CCO-N10. A continuación, se presentan los principales hallazgos por apartados para facilitar una mayor claridad expositiva.

\section{Descriptivos}

Los valores promedio de los items componentes oscilan entre una media mínima de 0,93 (item 8) y una máxima de

Tabla 1. Datos descriptivos, análisis factorial y fiabilidad de la versión abreviada del Cuestionario de Craving a Cocaína (CCO-N-10)

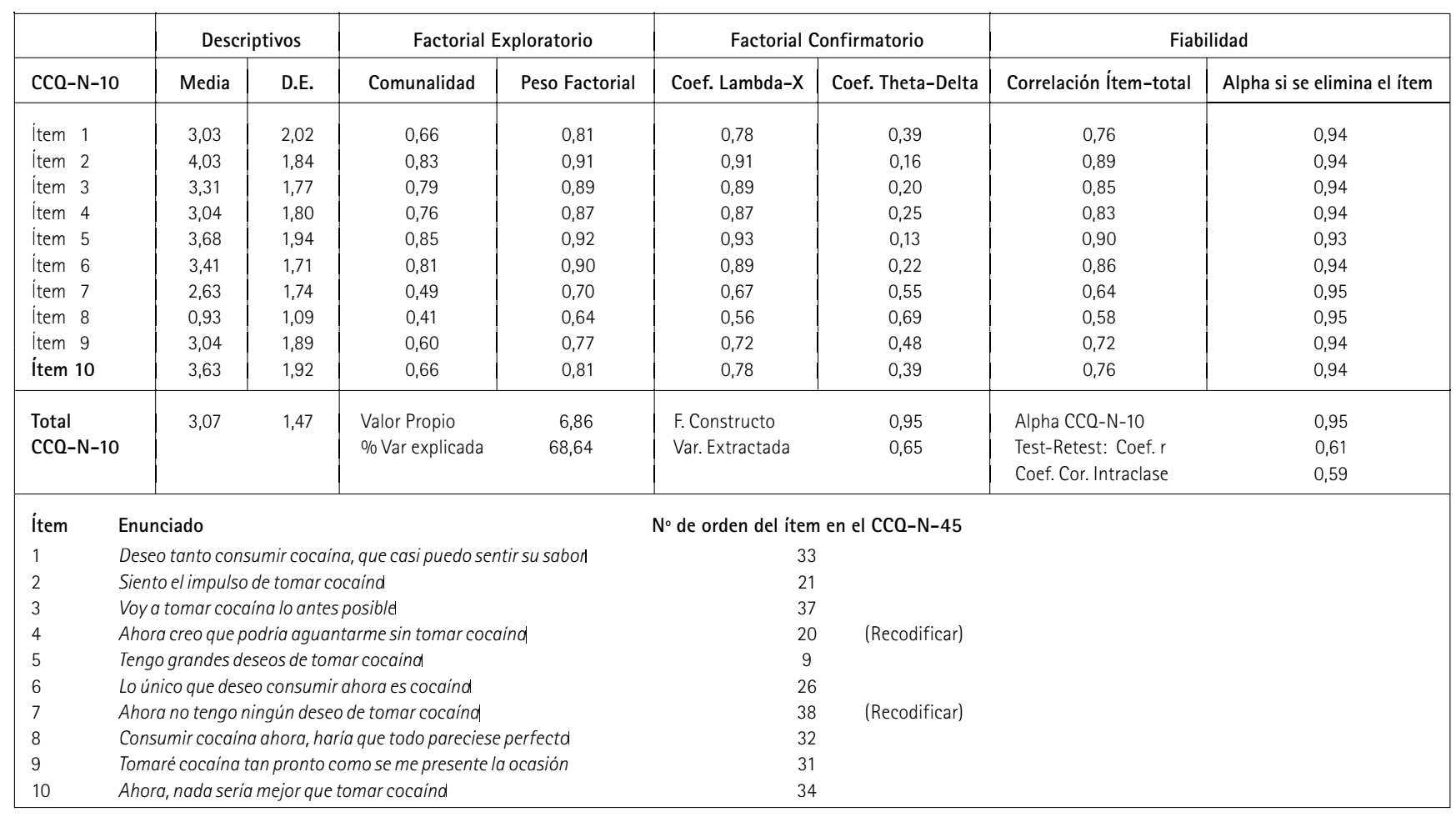


4,03 (item 2), siendo la Media (M) del índice sumario de 3,07, con una Desviación Estándar (DE) de 1,47 y un rango de valores que oscila entre los valores mínimo de 0,2 y máximo de 6 , en un recorrido posible de 0 a 6 .

\section{Fiabilidad}

La consistencia interna del CCQ-N-10 ha alcanzado un valor alpha de 0,947 y la retirada de ninguno de sus items permite mejorar dicho valor. Los valores de correlación de cada uno de los ítems con el total de la escala se sitúa en todos los casos por encima del valor 0,58, correspondiente en este caso al item 8. La fiabilidad test-retest proporciona un valor de correlación de Pearson de 0,61 y un Coeficiente de Correlación Intraclase de 0,59. Por otro lado, y anticipándonos a los resultados del análisis factorial confirmatorio, a partir de los coeficientes Lambda-X y Theta-Delta se ha obtenido una fiabilidad de constructo de 0,948 con un porcentaje de varianza extractada del $65,3 \%$.

\section{Validez de Constructo}

\section{Análisis Factorial Exploratorio (AFE)}

Previo al AFE del CCO-N-10, se realizó otro sobre la versión extendida del instrumento, el CCQ-N-45. Se obtuvo un valor de 0,71 en la prueba KMO y un valor de Ji cuadrado de 1234,83 ( $p<0,001)$ para la prueba de esfericidad de Bartlett, mostrando que la matriz de correlaciones resulta adecuada para su factorización. Se han obtenido cuatro factores, siendo sus valores propios de 6,$3 ; 4,0 ; 1,9$ y 1,4 , y su porcentaje de varianza explicada de $35,20 \% ; 22,46 \% ; 10,66 \%$ y $7,99 \%$, respectivamente. La fiabilidad alcanzada por el CCQ-N-45 ha resultado de un valor alpha de 0,88.

El AFE realizado sobre el CCQ-N-10 (Tabla 1), presenta valores óptimos para las pruebas $\operatorname{KMO}(0,85)$ y esfericidad de Bartlett $\left(\chi^{2}=622,5 ; p<0,001\right)$, obteniéndose un único factor que explica una varianza total del $68,6 \%$, con pesos factoriales por encima del valor 0,70 en todos los casos salvo para el ítem 8 que alcanza un valor de 0,64. Todas las comunalidades se sitúan por encima del valor de 0,40.

\section{Análisis Factorial Confirmatorio (AFC)}

Asimismo, el CCO-N-10 fue sometido a un AFC, presentándose los resultados en la Tabla 1. En dicha tabla, pueden observarse los pesos factoriales, representados por los coeficientes Lambda- $\mathrm{X}$, y los errores, especificados por los coeficientes Theta-Delta. Al igual que en el caso del AFE, los pesos factoriales son en general altos salvo en el caso del ítem 8 . Las pruebas de bondad de ajuste $\left(\chi_{(35)}^{2}=106 ; p<0,001 ; \chi^{2}\right)$ $\mathrm{gl}=3,03 ; \mathrm{CFI}=0,91 ; \mathrm{GFI}=0,75 ; \mathrm{RMSR}=0,06 ; \mathrm{RMSEA}=0,18$ ) muestran una falta de ajuste de los datos al modelo unifactorial propuesto. Dado este resultado, se buscó un mejor ajuste del modelo mediante la aplicación de las pruebas del contraste multiplicador de Lagrange y el contraste de Wald. El contraste de Wald sugiere no retirar ninguno de los parámetros existentes, mientras el contraste de Lagrange propone incorporar asociaciones entre los coeficientes ThetaDelta. Un intento por lanzar nuevos modelos a partir de estos contrastes se vio censurado dado el bajo número de efectivos de la muestra.

Tabla 2. Matriz de correlaciones de las versiones del CCQ-N y otros constructos afines. Análisis de la Validez Concurrente

\begin{tabular}{|c|c|c|c|c|}
\hline & \multicolumn{4}{|c|}{ Versiones del $\mathrm{CCO}-\mathrm{N}$} \\
\hline & Alpha & $\mathrm{CCO}-\mathrm{N}-10^{(1)}$ & $\mathrm{CCO}-\mathrm{N}-45^{(2)}$ & $\mathrm{CCO}-\mathrm{N}-35^{(3)}$ \\
\hline CCQ-N-45 - Cuestionario de Craving a Cocaína & 0,881 & $0,931^{* *}$ & & \\
\hline CCQ-N-35 - Cuestionario de Craving a Cocaina & 0,809 & $0,762^{* *}$ & $0,832^{* *}$ & \\
\hline EVA - Craving - Escala Visual Analógica & - & $0,657^{* *}$ & $0,821^{* *}$ & $0,730^{* *}$ \\
\hline CCS - Escala de Craving de Cocaina & 0,929 & $0,645^{*} a^{*}$ & $0,718^{* *}$ & $0,611^{* *}$ \\
\hline SDS - Escala de Severidad de la Dependencia & 0,796 & $0,527^{* *}$ & $0,629^{* *}$ & $0,508^{* *}$ \\
\hline ICP - Impresión Clínica Psiquiátrica & - & $0,502^{* *}$ & $0,515^{* *}$ & $0,499^{* *}$ \\
\hline ICG - Impresión Clínica Global & - & $0,343^{*}$ & $0,328 *$ & $0,307^{*}$ \\
\hline Años de consumo & - & 0,080 ns & $0,087 \mathrm{~ns}$ & 0,072 ns \\
\hline $\mathrm{N}^{\circ}$ de días de consumo último mes & - & $0,503^{* *}$ & $0,467^{* *}$ & $0,412^{* *}$ \\
\hline \multicolumn{5}{|c|}{$\begin{array}{l}\text { Nota.- (1) CCQ-N-10 - Versión abreviada de } 10 \text { items; (2) CCQ-N-45 - Versión extendida de } 45 \text { items o CCQ-Now; (3) CCQ-N-35 - Versión extendida de } 35 \text { ítems a la que se han } \\
\text { eliminado los items componentes del CCQ-N-10 para controlar el efecto de la autocorrelación. }\end{array}$} \\
\hline \multicolumn{5}{|l|}{ Valores de $p:{ }^{* *} p<0,001 ; \quad{ }^{*} p<0,01 ;$ ns no significativo } \\
\hline
\end{tabular}

\section{Validez Concurrente}

El análisis de concurrencia del CCQ-N-10 respecto a sus versiones extendidas (Tabla 2) ofrece coeficientes de correlación altos tanto con el CCQ-N-45 $\left(r=0,93 ; R^{2}=0,86\right)$ como con el
CCQ-N-35 $\left(r=0,76 ; R^{2}=0,58\right)$, siendo asimismo alta la correlación entre el CCQ-N-45 y CCO-N-35 $(r=0,83)$. El contraste de diferencia de medias entre las versiones abreviada ( $\mathrm{M}_{\mathrm{CCQ}-\mathrm{N}-10=}$ $3,07 ; \mathrm{DE}=1,47)$ y extendida $\left(\mathrm{M}_{\mathrm{CCO}-\mathrm{N}-45}=3,40 ; \mathrm{DE}=1,38\right)$ ha resultado estadísticamente significativo $(t=4,81 ; p<0,001)$. 


\section{Validez Discriminante}

El contraste de diferencia de medias obtenidas en el CCQ$\mathrm{N}-10$ entre las personas diagnosticadas de dependencia de cocaína $(M=3,62 ; D E=0,87)$ respecto de las que presentan un trastorno por abuso de cocaina $(M=2,76 ; D E=1,66)$ ha resultado estadisticamente significativo $\left(F_{(1 ; 60,6)}=7,16 ; p=0,010\right)$. De igual modo, en el caso del CCQ-N-45 el contraste de diferencias también resulta significativo $\left[\mathrm{M}_{\text {Dependencia }}=3,90(\mathrm{DE}=\right.$ $\left.0,90) ; M_{\text {Abuso }}=3,12(D E=1,53) ; F_{(1 ; 60,9)}=6,46 ; p=0,014\right]$.

\section{Validez Convergente}

Por último, en la Tabla 2 se presenta la matriz de correlaciones de las versiones de 10, 45 y 35 items del CCQ-N entre sí y de éstas respecto a otros constructos afines. La consistencia interna de las escalas utilizadas presentan, en todos los casos, coeficientes alpha de Cronbach por encima del valor 0,80 reflejando una alta precisión de medida.

El CCQ-N-10 correlaciona positiva y significativamente con otros constructos de valoración del craving (EVA-Craving y CCS), asi como con la severidad de la dependencia, la impresión clínica y el número de días de consumo durante el último mes (Tabla 2). No obstante, la asociación con el número de años de consumo no ha mostrado significación estadistica. Similar patrón de asociación se encuentra respecto a las versiones extendidas del CCO-N.

\section{DISCUSIÓN}

Con el presente estudio se han valorado la validez y fiabilidad de la versión abreviada de 10 items del Cuestionario de Craving a Cocaina, ofreciendo resultados que permiten concluir que la versión adaptada al español del CCO-N-10 presenta una óptima adecuación métrica. Al igual que en los estudios de adaptación anglosajones ${ }^{17-18}$, el CCO-N-10 correlaciona fuertemente con la versión extendida de 45 items (CCQ-N-45), explicando hasta un $86 \%$ de su varianza, e incluso mantiene una fuerte asociación $(r=0,76)$ con la escala extendida cuando se eliminan los items que constituyen la escala abreviada. La versión española del CCQ-N-10 presenta asimismo una alta consistencia interna con un valor próximo a la unidad (alpha de Cronbach $=0,95$ ) y ofrece coeficientes de asociación moderados-altos con constructos afines que dan razón de una adecuada validez convergente.

En el ámbito clínico el tiempo dedicado a cada paciente suele ser escaso para el conjunto de acciones que se deben desarrollar en cada sesión (p.ej., valoración del estado-situación del paciente, revisión de tareas programadas, aplicación terapéutica, etc.), por lo que de procederse a una evaluación sistemática suelen preferirse escalas de valoración breves. En muchos de los casos se recurren a escalas visuales-analógicas (EVA), siendo algo muy habitual en la valoración de craving. No obstante, las EVA están limitadas a un único enunciado (escalas de ítem único) de modo que presentan una mayor varianza de error que los instrumentos multi-items ${ }^{27}$. Por otro lado, los instrumentos de ítem único asumen que los sujetos respondientes definen y entienden el concepto valorado del mismo modo, lo cual no siempre es una asunción aceptable ${ }^{9}$. El CCQ-N-10 presenta la ventaja de ser un instrumento breve respecto a las versiones extendidas $y$, si bien se tarda algo más en responder que una EVA, permite garantizar una mayor homogeneidad en la valoración y comprensión del constructo valorado, ya que sus diez items dan cuenta de las diferentes dimensiones teóricas que constituyen el concepto de craving, lo que ofrece una mayor sensibilidad de medida y captación de los cambios.

Psicométricamente, el CCQ- $\mathrm{N}-10$ respecto a la versión extendida presenta una serie de caracteristicas que lo convierten en un instrumento muy atractivo en el ámbito clínico. En primer lugar, consigue explicar hasta el $86 \%$ de la varianza del CCQ-N-45, por lo que reproduce suficientemente los resultados que aquel pudiera proporcionar. No obstante, el CCO-N10 tiende a infraestimar la valoración promedio de intensidad del craving, lo cual podria tener repercusiones a nivel clínico. Ahora bien, también cabe plantearse que no sea el CCQ-N10 el que subestima la valoración, sino el CCQ-N-45 quien la sobreestima. Recordemos que la versión extendida del CCO valora hasta cinco dimensiones teóricas del craving, alguna de las cuales puede ofrecer puntuaciones medias que difieran significativamente del indice total promedio, afectando a éste hacia un incremento de su puntuación. Sería preciso en subsiguientes análisis comprobar este supuesto y en tal caso podria ser conveniente utilizar el CCQ-N-10 como un índice global para su aplicación en el ámbito clínico, y reservar el CCO-N-45 desglosado en sus dimensiones o factores para su utilización en investigación.

La consistencia interna del CCQ-N-10 (alpha $=0,95)$ es mayor que la del CCO-N-45 (alpha $=0,88$ ), y su fiabilidad de constructo $(0,95)$ y su varianza extractada $(65,3 \%)$ verifican su fuerte precisión de medida. Por otro lado, su fiabilidad de reproducción estimada por la prueba test-retest $(\mathrm{CCl}=0,58)$ resulta, si no óptima, si al menos moderada. Una posible explicación de este resultado podría achacarse a que se trata de una muestra clínica que inicia tratamiento por sus problemas con la cocaina, y aunque apenas pasan entre dos y tres semanas desde la valoración inicial, en el retest podrian estar reflejándose cambios de distinto grado en los sujetos que repercutirian en la varianza de las estimaciones $y$, por tanto, en su fiabilidad de reproductibilidad. Hipotéticamente, una muestra no sometida a tratamiento (en consumo continuado) o que ya haya alcanzado los objetivos del tratamiento (en abstinencia), mantendría el mismo nivel de intensidad del craving, o al menos sufriría menos variaciones en periodos cortos de tiempo, de modo que la aplicación del instrumento en dicha situación reportaría una mayor estabilidad de medida temporal.

Respecto a su validez de constructo, al igual que hallaron Tiffany et al..$^{9}$ en la factorización de la versión extendida del $\mathrm{CCQ}-\mathrm{N}$, el primer factor obtenido en nuestro estudio agrupa los items del CCQ-N-10. El AFE sobre los diez items del CCQ$\mathrm{N}-10$ presenta unidimensionalidad y sólo el item 8 presenta un comportamiento algo dispar a la homogeneidad del resto de items, aunque no por ello pierde relación con el constructo global. Por tanto, tomando en consideración los datos del análisis 
exploratorio podríamos proponer la unidimensionalidad del CCQ-N-10 y su factibilidad para extraer una puntuación única del constructo. No obstante, el análisis factorial confirmatorio afina su interpretación y propone la existencia de un efecto de respuesta diferencial entre los ítems componentes. Es decir, el AFC no contradice la unidimensionalidad del constructo, pero plantea la existencia de algunos patrones de respuesta de los items que requeriria un análisis más pormenorizado. Si bien contamos con un número limitado de casos para realizar este análisis con precisión, una primera aproximación derivada de las relaciones observadas entre los coeficientes Theta-Delta del AFC muestra como los items con enunciados orientados de forma activa hacia el deseo de consumo conforman un patrón de respuesta, y los enunciados orientados hacia el control del consumo forman otro patrón. Esto es, por ejemplo, los sujetos que puntúan alto en control del consumo (p.ej: Ahora creo que podría aguantarme sin tomar cocaína) tienden a puntuar bajo en los items que denotan deseo o falta de control (p.ej.: Siento el impulso de tomar cocaína).

Por otra parte, los resultados de validez convergente muestran como altas puntuaciones en el CCQ-N-10, es decir, una mayor intensidad del craving, se asocia con un mayor número de días de consumo de cocaina en el mes previo, a un mayor grado de severidad de la adicción y a una mayor gravedad en la impresión clínica -de mayor efecto en el área de valoración psiquiátrica que en el área general-. Es decir, la valoración del craving a través del CCQ-N-10 muestra consistencia con otros constructos afines del área de valoración clínica y permite apreciar y medir el grado de severidad o compromiso del trastorno adictivo en el espacio de intervención terapéutica. En tanto que se trata de un instrumento autoaplicado, ajeno a las interferencias o valoraciones del clínico, el CCO-N-10 puede utilizarse como herramienta para la homogeneización de resultados clínicos cuando se evalúan sujetos pertenecientes a distintos espacios de tratamiento.

Asimismo, el CCO-N-10 permite diferenciar el grado de intensidad del craving entre personas que reciben distintos diagnósticos por su problema de consumo de cocaina. Las personas diagnosticas de dependencia, por definición con un grado mayor de compromiso o severidad en su trastorno adictivo, presentan de forma estadisticamente significativa una mayor puntuación en el CCO-10 que las personas diagnosticadas de abuso de cocaina, es decir, presentan una mayor intensidad del craving.

A pesar de que los resultados del presente estudio alientan a considerar el CCO-N-10 como un instrumento válido y fiable, hemos de ser cautelosos respecto a su alcance y capacidad conclusiva dadas las limitaciones metodológicas del estudio. En primer lugar, cabe señalar el limitado tamaño de la muestra. Muestras con mayor número de casos contribuirian a reducir el error de medida y con ello aumentar su precisión. No obstante, ha de observarse que para el restringido número de efectivos utilizados en el presente estudio de validación se han obtenido coeficientes considerablemente altos, y de la inclusión de más sujetos cabría esperar una mejora de dichos indices. Asimismo, un aumento de la muestra permitiría obtener una mayor estabilidad en los modelos probados a través del análisis factorial confirmatorio. Otra de las limitaciones, ya apuntada por el grupo de Sussner ${ }^{17}$, es la utilización de la versión extendida del CCO-N para la validación concurrente de la versión abrevia$d a$, en tanto que los diez ítems componentes de esta última se hallan comprendidos en la primera $y$, por tanto, se produce un efecto de auto-correlación que desvirtúa el verdadero efecto de estimación. No obstante, cuando se prescinde los diez items del CCQ-N-10 en la versión extendida (CCQ-N-35) y se valora el grado de asociación, se encuentra una relación estadísticamente significativa y de magnitud relevante $(r=0,76)$, lo que permite concluir que el CCO-N-10 ofrece una medición adecuada del concepto de craving.

Por otra parte, falta por valorar las características de la versión breve del CCO-N en lo que respecta a su validez predictiva, sensibilidad al cambio y estimación del punto de corte a partir del cual la intensidad del craving puede considerarse clínicamente significativa. Para ello se precisan nuevos estudios que utilicen muestras clínicas de mayor número de efectivos y diseños longitudinales suficientemente dilatados para la estimación de los cambios. Tareas estas que habrán de abordarse en un futuro, esperemos próximo.

\section{CONCLUSIONES}

Son varias las características que sugieren que el CCO-N10 puede convertirse en un instrumento atractivo en el ámbito clínico para la valoración del craving. En primer lugar, su brevedad y legibilidad; es un instrumento que no tarda en administrarse más de tres minutos y sus items son fácilmente comprendidos por los sujetos en tratamiento. En segundo lugar, se muestra como un instrumento con precisión de medida, lo que permite garantizar que mide consistentemente lo que pretende medir. En tercer lugar, presenta validez de constructo y concurrente, lo que garantiza que mide aquello que pretende medir (la intensidad del craving) y lo mide de forma pareja a como lo hacen sus versiones extendidas $u$ otros instrumentos afines. A este respecto, el CCQ-N-10 reproduce de forma válida los resultados que se obtendrian con el CCQ-N45 y, por otra parte, da razón de un constructo más compacto sobre el craving del que puede ofrecer una escala analógica de ítem único. Todo ello permite presentar el CCO-N-10 como un instrumento a tener en cuenta en la valoración de la intensidad del craving en el ámbito clínico. $Y$, en cuarto lugar, presenta validez discriminante, ya que permite diferenciar la intensidad del craving en grupos diagnósticos que por definición (DSMIV) difieren en el grado de intensidad de la severidad de la adicción: abuso vs dependencia de cocaína.

Ahora bien, como advierten Sussner et al. ${ }^{17}$, previa a la elección de la versión breve del $\mathrm{CCO}$, clínicos e investigadores deben considerar cuidadosamente cuáles son sus intereses en el proceso de evaluación. A diferencia de la versión extendida del CCQ-N (CCQ-N-45), que es una medida multidimensional de los componentes teóricos del craving ${ }^{9,12-14}$, el CCO-N-10 ha sido desarrollado para ofrecer una medida global de la intensidad del craving a cocaina. En otras palabras, no está destinado para valorar los componentes independientes tales como la anticipación de los efectos positivos de consumir o la carencia de control sobre el uso de cocaina. Por tanto, el CCQ-N-10 ha 
de utilizarse sólo cuando la valoración del craving global sea la medida de interés, y esto sucede en el campo de la valoración clínica y en la evaluación de resultados terapéuticos ${ }^{3}$.

\section{REFERENCIAS}

1. UNDCP and WHO. Informal Expert Committee on the drugcraving mechanism. Wien: United Nations International Drug Control Programme and World Health Organization, Technical Report Series (n V 92-54439 T), 1992.

2. Pérez de los Cobos J, Santis R, Tejero A. La medición del craving de sustancias psicoactivas ilegales. Trastor Adict 1999; 1: 22-6.

3. Iraurgi I, Corcuera N. Craving: Concepto, medición y terapéutica. Norte - Salud Mental 2008; 32: 9-22.

4. Crits-Christoph P, Siqueland L, Blaine J, Frank A, Luborsky L, Onken LS, Muenz L, Thase ME, Weiss RD, Gastfriend DR, Woody G, Barber JP, Butler SF, Daley D, Bishop S, Najavits LM, Lis J, Mercer D, Griffin ML, Moras K, Beck AT. The National Institute on Drug Abuse Collaborative Cocaine Treatment Study: rationale and methods. Arch Gen Psychiatry 1997; 54: 721-6

5. Ramos J, Tejero A, Trujols J. ¿Craving como elemento central o como epifenómeno de las conductas adictivas? Una revisión de la propuesta de Tiffany. Adicciones 1998; 10: 217-21.

6. Drummond DC. Conceptualizing addiction: theories of drug craving, ancient and modern. Addiction 2001; 96: 33-46.

7. Tejero A, Trujols J, Siñol N. Evaluación del craving de cocaína. En: Pérez de los Cobos J, Tejero A, Trujols J, editores. Instrumentos clínicos para la evaluación de la dependencia de la cocaína. Barcelona: Ars Médica; 2003, p. 23-50.

8. Voris J, Elder I, Sebastián PA. A simple test of cocaine craving and related responses. J Clin Psychol 1991; 47: 320-3.

9. Tiffany ST, Singlenton E, Haertzen CA, Henningfield IE. The development of a cocaine craving questionnaire. Drug Alcohol Depend 1993; 34: 19-28.

10. Weiss RD, Griffin ML, Hufford C. Craving in hospitalized cocaine abusers as a predictor of outcome. Am J Drug Alcohol Abuse 1995; 21: 289-301.

11. Weiss RD, Griffin ML, Hufford C, Muenz LR, Najavits LM, Jansson $S B$, et al. Early prediction of initiation of abstinence from cocaine: use of a craving questionnaire. Am J Addict 1997; 6: 224-31.

12. Tiffany ST. A cognitive model of drug urges and drug-use behavior: Role of automatic and nonautomatic processes. Psychol Rev 1990; 97: 147-68.

13. Tiffany ST. Cognitive concepts of craving. Alcohol Res Health 1999; 23: 215-24.
14. Tiffany ST, Conklin CA. Cognitive processing model of alcohol craving and compulsive alcohol use. Addiction 2000; 95 (supl. 2): 145-53.

15. Muñoz-Garcia MA. Mecanismos motivacionales del craving: Ansia por el tabaco. Tesis doctoral. Granada: Universidad de Granada; 2008.

16. Muñoz-Garcia MA, Martínez JA, Tejero A, Cepeda-Benito A. Development of the brief Spanish Craving QuestionnaireGeneral. Psicothema 2008; 20: 545-50.

17. Sussner BD, Smelson DA, Rodrigues $S$, Kline A, Losonczy $M$, Ziedonis $D$. The validity and reliability of a brief measure of cocaine craving. Drug Alcohol Depend 2006; 83: 233-7.

18. Paliwal P, Hyman SM, Sinha R. Craving predicts time to cocaine relapse: Further validation of the Now and Brief version of the cocaine craving questionnaire. Drug Alcohol Depend 2008; 93: 252-9.

19. Landabaso M, Iraurgi I, Aizpuru A, Caso C, García-San-Cornelio J, García-Serrano G, et al.. Uso del Topiramato en el tratamiento de la abstinencia a cocaina y alcohol: Un estudio naturalístico con seguimiento de 6 meses. Póster presentado en el $X$ Congreso Nacional de Psiquiatría. Sevilla, 16-21 de Octubre de 2006.

20. Gossop M, Darke S, Griffiths P, Hando J, Powis B, Hall W, Strang J. The Severity of Dependence Scale (SDS): psychometric properties of the SDS in English and Australian samples of heroin, cocaine and amphetamine users. Addiction 1995; 90: 607-14.

21. Gossop M, Best D, Marsden J, Strang J. Test-retest reliability of the Severity of Dependence Scale. Addiction 1997; 92: 353-4.

22. González-Saiz F, Salvador-Carulla L. Estudio de fiabilidad y validez de la versión española de la escala severity of dependence scale (SDS). Adicciones 1998; 10: 223-32.

23. Iraurgi I, Lozano 0, González-Saiz F, Trujols J. Valoración psicométrica de la Escala de Dependencia de Sustancias (SDS) a partir de dos modelos de análisis: la Teoría Clásica de los Test (TCT) y la Teoria de Respuesta al İtem (TRI). Bol Psicol 2008; 93: 41-57.

24. Guy W. (ed). ECDEU Assessment Manual for Psychopharmacology. Rockville, MD: US Department of Heath, Education, and Welfare Public Health Service Alcohol, Drug Abuse, and Mental Health Administration; 1976.

25. Busner J, Targum SD. The Clinical Global Impressions Scale: Applying a research tool in clinical practice. Psychiatry MMC, http://psychiatrymmc.com, 2007.

26. Cea MA. Análisis multivariable. Teoria y práctica en la investigación social. Madrid: Editorial Sintesis; 2002.

27. Sayette MA, Shiffman S, Tiffany ST, Niaura RS, Martin CS, Shadel WG. The measurement of drug craving. Addiction 2000; 95 (Suppl. 2): S189-S210. 